\title{
HISTORIA DE UNA \\ PERMANENCIA. LAS ELITES DE \\ SANTIAGO DE CHILE EN \\ EL SIGLO XVIII: \\ FAMILIA Y PODER LOCAL
}

\author{
Jean-Paul Zúñiga \\ Université de Reims
}

Todo estudioso que se interese en la estructura social de las sociedades de la América española colonial, se ve rápidamente confrontado con la representación tradicional de los diversos elementos que la componían, representación en la que a cada grupo, en función de su adscripción "étnica", le correspondía un determinado lugar en la organización social colonial. Así, los Españoles -y sus descendientes criollos-constituían la cima de la pirámide social, dominando a todos los otros grupos, indios, mestizos, negros y mulatos.

De cierta manera, esta visión "racialista" no parece sino la perpetuación del modelo teórico de las dos "repúblicas", según el cual estaba organizado el 
imperio, al que se ha añadido la población de origen africano cuyo estatuto servil la relegaba a una posición específica. Esta jerarquización en categorías étnicas, a pesar de que coincida en alguna manera -muy esquemática- con la realidad social, parece un modelo analítico muy poco satisfactorio, ya que no ayuda mucho a entender la construcción y la evolución de las sociedades fundadas por la colonización española.

Lo interesante es constatar que esta representación, comúnmente utilizada para estigmatizar el carácter inicuo del sistema colonial, es la misma que durante mucho tiempo dio su legitimidad a las elites coloniales, quienes la reivindicaban. Precisamente por que no eran de la misma naturaleza que la oscura plebe les coiTespondía tener las riendas de la "república". A pesar de la escasa utilidad de esta visión un tanto maniquea de las sociedades coloniales -y de la abundante producción historiográfica sobre la relatividad y complejidad de las clasificaciones etno-sociales coloniales- ${ }^{1}$ estas separaciones en categorías "étnicas" siguen funcionando en gran medida, de manera reificada, influyendo incluso en la delimitación de competencias científicas; es de ésta forma que el mundo criollo suele ser el campo reservado a los historiadores "duros", mientras que el mundo indígena, la "República de los Indios" es ampliamente tomada a cargo por los etnohistoriadores venidos de la antropología. ${ }^{2}$ La separación teórica en castas de las sociedades coloniales continúa así siendo operacional.

En el caso de Chile esta manera de enfocar el período colonial ha tenido una fortuna historiográfica particularmente larga. Así, la historia social de Chile durante la época colonial estaría caracterizada por un fenómeno "doble" de reproducción de las elites y de los grupos subalternos siguiendo patrones demográficos diferentes -y específicos a cada uno de estos dos conjuntos- produciendo lo que algunos

1 Sin hablar del trabajo clásico de Magnus Mómer, es preciso mencionar entre otros los trabajos de Jonathan I. Israel, Race. Class and Politics in Colonial Mexico, 1610-1670, Londres, Oxford UP, 1975; de Patricia Seed, en particular "Social Dimensions of Race: México, 1753",Hispanic American Historical Review, vol. 62, No. 4, 1982; o de Martin Minchom, The People of Quito, 1690-1810. Change and Unrest in the Underclass, Bouldcr-San Francisco-Oxford, Westview Press, 1994.

2 A tal punto que para una cierta historiografía estadounidense, una "deshispanización" total es un préaiabaie para el investigador y para sus fuentes si quiere hacer una "verdadera" historia de los pueblos indígenas durante el período colonial. Ver Matthew Restall, The Maya World. Yucatec Culture and Society, 1550-1850, Stanford, Stanford University Press, 1997. 
historiadores chilenos decimonónicos, e incluso del siglo XX, trataban de presentar como dos pueblos y una nación. Aunque estos términos, demasiado explícitos, nunca fueran usados abiertamente, el concepto es fuertemente sugerido precisamente por los historiadores que provenían o se relacionaban con las elites sociales. En este sentido, el caso de Domingo Amunátegui Solar y su Historia social de Chile, publicada en 1932, es paradigmático. En efecto, su obra consta de dos grandes partes ("El pueblo" y "La aristocracia"), y cada una de estas partes no es sino la narración de la génesis paralela -física y cultural- de dos grupos. ${ }^{3}$ Es asimismo particularmente revelador que el historiador chileno Tomás Thayer Ojeda, en su minuciosa obra biográfica Formación de la sociedad chilena y censo de la población de Chile, no reparase que a su censo le faltaba -aunque sólo fuese bajo forma de una mera alusión o de cálculo global con fines metodológicos- la población aborigen (y los escasos africanos)... es decir más del 98\% de la población del primer Chile colonial. ${ }^{4}$ Ya sea abiertamente o por omisión, el resultado es semejante: la separación en dos grupos fundamentalmente diferentes y que parecen, por así decirlo, herméticos el uno al otro.

Lo que se desprende de esta visión -de estas visiones- es que el desarrollo paralelo de estos grupos no habría seguido las mismas pautas ni producido los mismos resultados. Así, los grupos subalternos se caracterizarían por lo que se podría llamar una cierta "estabilidad", pues al grupo primigenio y básico del indígena y del mestizo sumarían con el correr del tiempo, y en proporciones variables, elementos africanos y de "españoles pobres", que engrosaron sus rangos sin cambiar fundamentalmente su origen y características.

Los grupos de la elite, por el contrario, son tradicionalmente vistos como inestables, inestabilidad social expresada por una sucesión de diferentes grupos en la cúspide político-económica de la sociedad chilena -pero que tenían todos, eso sí, como denominador común ser españoles- grupos que de Vicuña

${ }^{3}$ Domingo Amunátegui Solar Historia social de Chile, Santiago, Ed. Nascimento, 1932.

${ }^{4}$ Tomás Thayer Ojeda. Formación de ja sociedad chilena y censo de la población de Chile en los años de 1640 a 1665, con datos estadísticos, biográficos, étnicos y demográficos, Santiago, Prensas de la Universidad de Chile, 1939. 
Mackenna a nuestros días se presentan como tres. El primero sería el de los conquistadores, grupo en el que los hombres de las regiones del sur de la corona de Castilla (Extremadura, Andalucía) constituían la mayoría. El segundo sería el de la ola de pobladores y administradores que llegan una vez que la tierra está conquistada (por lo menos nominal mente) y que también es designado como grupo "castellano" sin más precisión, y que está cronológicamente ubicado en el siglo XVII. En fin, el tercer grupo, llamado a permanecer y a fundar -por fin- una "'verdadera" elite que se perpetuaría hasta la independencia y más allá, sería el de los "Vascos"5, emigrantes desde fines del siglo XVII pero sobre todo en el siglo XVIII, cuya imbricación con el mundo del comercio y por ende con el mundo "moderno" habría sido la herramienta de una "victoria social" sancionada justamente por su permanencia en las cimas del poder. Declinada de todo tipo de maneras, desde una interpretación racista -producto de una curiosa mezcla de provincialismo y de literatura histórica y antropológica mal digerida- ${ }^{6}$ hasta una vertiente moderna más atenta a lo "social", esta sucesión, este reemplazo de las elites no ha sido esencialmente cuestionado. ${ }^{7}$

Es preciso añadir aquí que este tema de la génesis separada -cualquiera que sea su grado de "realidad" o de construcción aposteriori- no es único al caso chileno, y muchos paralelos pueden ser establecidos con otras regiones de la antigua América española. Sin embargo, su boga historiográfica -bajo diferentes formasen otras palabras su longevidad, es debida a que la aserción de un desarrollo paralelo del "pueblo" y de la elite reposa en la constatación efectiva de la existencia de toda una serie de estrategias de distinción utilizadas por los estratos altos de la

\footnotetext{
${ }_{5}^{5}$ Término que en realidad cubre también a Navarros y a hombres de la cornisa cantábrica en general. $\quad{ }^{6} \quad$ Esta actitud, comprensible en historiadores como Luis Thayer Ojeda o incluso en el mismo Benjamín Vicuña Mackenna, hombres del siglo XIX, lo es mucho menos en historiadores del siglo XX como Francisco A. Encina. Ver, F. A. Encina, Historia de Chile desde la prehistoria hasta 1891, Santiago, Ed. Nascimento, 1944.

${ }^{7}$ Efectivamente de Vicuña Mackenna a Francisco Encina, ésta era la manera de presentar la historia de los estratos más altos, pero de cierta forma, historiadores aún más recientes postulan esta inestabilidad "estructural" de las elites. Ver en particular Mario Góngora, Encomenderos y estancieros. Estudios acerca de la constitución social aristocrática de Chile después de la conquista. 1580-1660. Santiago, Universidad de Chile, 1970; y Armando de Ramón, Santiago de Chile. 1541-1991. Historia de una sociedad urbana, Madrid, MAPFRE, 1992. Por otro lado, la idea de una separación de la sociedad chilena colonial en grupos étnicos estancos, parece correr en filigrana en el trabajo de Alvaro Jara. Los asientos de trabajo y la provisión de mano de obra para los no-encomenderos en la ciudad de Santiago, 1586-1600. Santiago, Universidad de Chile. 1959: y de manera mucho más explícita en la obra de Armando de Ramón ya citada (que resume por otra parte sus trabajos anteriores con el mismo enfoque).
} 
sociedad chilena -fenómeno común a todo el mundo colonial- y que es bien conocido por todos los historiadores familiarizados con el comportamiento de las elites criollas. ${ }^{8}$ En estas estrategias, el comportamiento matrimonial de la oligarquía juega un papel de primera importancia. La paradoja es que estas actitudes, cuya finalidad no era sino la permanencia, sirvan de base, en el caso chileno, para definir a un estrato cuya característica sería precisamente la inestabilidad.

Esta contribución, por consiguiente, tiene por objeto interrogarse sobre las prácticas de distinción y de alianza considerando en qué medida éstas explican las peculiaridades de la elite chilena del siglo XVIII. En otras palabras, ¿̇existe o no una ruptura en estas prácticas durante el siglo XVIII que permita hablar del auge de nuevos grupos sociales que reemplacen a las antiguas elites? A través de un estudio somero de los notables locales -considerados a través de su figuración como ediles del cabildo de Santiago- de sus prácticas matrimoniales y patrimoniales se tratará de demostrar cómo lo que aparece como una sucesión de diferentes grupos y redes es un espejismo, que oculta una extraordinaria estabilidad de las elites coloniales, estabilidad que quizás sea una de las principales particularidades del Chile colonial, con un peso considerable para entender incluso su historia independiente.

La manera tradicional de mostrar este movimiento de reemplazo de las elites al cual se viene haciendo alusión ha consistido siempre -para simplificar- en tratar de examinar el devenir de los troncos familiares hispánicos fundadores de la sociedad colonial, tarea efectuada las más de las veces gracias al estudio de las grandes familias que dominaban la vida local en diferentes periodos de la historia de Santiago de Chile. ${ }^{9}$

8 En particular el estudio clásico de David Brading, Mineros y comerciantes en el México borbónico, 1763-1810. México, Fondo de Cultura Económica, 1975; Susan M. Socolow, "Marriage, Birth, and Inheritance: The Merchants of Eighteenth-Century Buenos Aires". Híspanic American Historical Review, vol. 60, No. 3, 1980: Diana Balmori el al., Notable Family Networks in Latin America, Chicago. University of Chicago Press, 1984; Louisa Shell Hoberman, Mexico 's Merchant Elite. 1590-/660, Durham, Duke UP, 1991; Michel Bertrand, "Du bon usagedes solidantes: étude du lacteur fámilial dans $l$ ' administraron des finances de Nouveile-Espagne", Robert Descimon et al., La figure historique de l'administrateur. Institutions. réseaux. pouvoirs en Espagne. en France et ait Portugal. XVle-XIXe siécles, París. Editions de l' EHESS, 1997.

9 Método de Mario Góngora. Encomenderos y estancieros, pero también de Arnold Bauer, Chilean rural society from the Spanish conques/ to 1930. Cambridge, Cambridge UP, 1975 y de Armando de Ramón, Santiago de Chile. 1541-1991. pero antes de ellos de la tradición historiográfica chilena de fines del XIX y comienzos del $\mathrm{XX}$. 
Qué se entiende por "dominación de la vida local"? Bajo este término se esconde toda una serie de criterios económicos y sociales cuya conjunción en un individuo o grupo de individuos constituye el ideal considerado por todos los contemporáneos como la cima de la jerarquía social y que conllevaba el reconocimiento expresado por el término "nobleza". ${ }^{10}$ Estos criterios son esencialmente la posesión de tierras, de medios financieros -lo que implica acceso tanto a la moneda metálica como al crédito-, de mano de obra encomendada, esclava o asalariada ${ }^{1_{1}}$ para poner en valor sus bienes raíces; de contactos con el mercado interno y exterior para poder dar salida a la producción agrícola que es durante todo el período colonial y después de la independencia, la base de la economía chilena. Es evidente que se tiene que agregar a todos estos criterios un último, fundamental, que es el ejercicio de algún tipo de poder político, ${ }^{12}$ dispensador tanto de poder factual como de prestigio.

En la época colonial, el núcleo por excelencia en donde se desarrollaba buena parte del poder local era el cabildo, y esto es así a nivel de todo el continente y cualquiera que fuere el grado de ruralización de las elites económicas. El protagonismo jugado durante el proceso de independencia por la ciudad como unidad política, esto es, a través de la emanación de la comunidad urbana que era el cabildo, ${ }^{13}$ no conoce ninguna excepción en el caso chileno. Es en el marco municipal que se gestan los primeros esbozos de la república independiente: este papel es así de importante pues sólo a esta institución se le reconocía un papel de representación de la comunidad (y no a la Audiencia o a cualquier otro tipo de institución colonial). Es evidente que esta función no surge ex nihilo con el siglo XIX. La importancia simbólica y el poder factual ejercido a través del cabildo son una realidad que atraviesa todos los siglos coloniales.

\footnotetext{
${ }^{10}$ Cf. Jean-Paul Zuniga, Espagnols d'outre-mer, tesis del Instituto Universitario Europeo de Florencia / EHESS, de próxima publicación (París, Éditions de l'EHESS).

${ }^{11}$ La importancia relativa de cada una de estas fuentes de fuerza de trabajo varía, por supuesto, dependiendo de las épocas: si la encomienda caracteriza el primer período colonial, ya desde el siglo XVII su importancia es más simbólica, de prestigio, que económica. Ver Mario Góngora. Encomenderos y estancieros.

${ }^{12}$ En esto hay coincidencia con la definición que daba Brian Loveman, Chile. The legacy of Híspanic Capitalism, New York, Oxford UP, 1979, p.78, en donde definía al poder político como el "bien" más importante en todos los períodos.

${ }^{13}$ Sobre este punto fundamental ver Geneviéve Verdo, Les provinces "désunies" du Río de la Plata: souveraineté et représentation politique dans l' indépendance argentine, 1808-1821, tesis de la Universidad de París I, 1998, 2 vol.
} 
Enfocando esta cuestión desde otro punto de vista, es posible constatar, en los tres siglos de colonia, que los miembros de las elites económicas, las familias que expresan su poder y prestigio por medio de títulos y hábitos de las órdenes militares, por funciones importantes en la administración del imperio (gobiernos, tribunales, obispados) son las mismas cuyos miembros pueblan la institución municipal, y esto es un hecho que se puede verificar del siglo XVI al XVIII.

Así, las familias de la Cerda, Toro Mazote, Irarrazabal, Larrain, Lecaros, Aguirre, Ruiz de Tagle, Prado y Covarrubias, Toro Zambrano, todas las cuales fundaron mayorazgos durante el siglo XVIII, figuran en buen lugar entre los ediles de la ciudad de Santiago durante todo el siglo. La misma constatación puede ser hecha con respecto a las familias que adquirieron títulos de Castilla, cuyos miembros permanecen ligados al cabildo: en el siglo XVIII, como en el XVI y el XVII, el cabildo es un observatorio irremplazable de las elites coloniales.

Ahora bien, volviendo a considerar el punto de partida -la medida por el apellido del movimiento de reemplazo de las elites antiguas por hombres nuevos-se puede decir que un examen rápido de la institución municipal tiende efectivamente a corroborar la idea de una gran fluidez de las elites, que parecen fugaces hasta fines del siglo XVII.

En efecto, si se analizan las listas de ediles, regidores y alcaldes, o de corregidores de Santiago y su jurisdicción, se encuentra efectivamente con que los nombres de los primeros años (Francisco de Riveros; Pedro de Miranda, Alonso de Córdoba, Diego García de Cáceres, Juan Jofré...) que evocan irresistiblemente la conquista, son reemplazados por "hombres nuevos" -para usar el término de Mario Góngora- durante el siglo XVII: Jerónimo de Molina, Domingo García Corvalán, mercaderes como Alonso del Campo Lantadilla, los hermanos Francisco y Miguel Ortiz de Elguea o Ignacio de la Carrera. Estos "hombres nuevos" son desplazados a su vez por una "ola" vasco-navarra a partir de finales del XVII, ola de la que proceden Juan de Lecaros, Tomás Ruiz de Azúa, Juan Felipe Martínez de Aldunate, Santiago de Larrain... y tantos otros hombres, fundadores o no de vínculos y mayorazgos, 
cuyos apellidos guardan hasta el día de hoy -la sociedad chilena conserva un marcado gusto por la memoria genealógica- ${ }^{14}$ el prestigio de más de dos siglos de figuración social.

Si Mario Góngora movilizó para su demostración de la aparición de nuevos hombres un conjunto documental detallado y expuso de manera precisa su metodología, ${ }^{15}$ la mutación de fines del siglo XVII y del siglo XVIII, por la visibilidad tan característica de la antroponimia vasca, no parece haber tenido un estudio tan detallado, ya que los apellidos vascos dan la impresión de bastarse a sí mismos para demostrar la importancia que tomó este grupo en los puestos políticos (corregimientos, cabildos), de justicia o en el comercio.

Y de hecho, si miramos la lista de los 182 individuos diferentes que ocupan los cargos de alcaldes y corregidores de Santiago de Chile entre 1690 y 1790 (muchos de los cuales lo hacen en repetidas ocasiones), no sólo los apellidos de los conquistadores han desaparecido casi completamente, sino que la importancia relativa de los antropónimos de origen vasco -más de 30 por ciento de los cabildantes tienen un apellido vasco o navarro- parece acreditar esa exagerada y muy conocida afirmación atribuida a Miguel de Unamuno según la cual los vascos habrían llevado a cabo dos grandes empresas en la historia, la Compañía de Jesús y Chile...

Y sin embargo, esta impresión de movimiento, que de cierta manera es efectivo, esconde una realidad más compleja, en la que la fluidez social aparente y su consiguiente reemplazo de las elites es, al mismo tiempo, para la vieja oligarquía, la única manera viable de perpetuarse, en un proceso que no tiene solución de continuidad.

La mejor manera de entender esta afirmación es examinar el comportamiento de los "elementos nuevos" dentro del cabildo de Santiago en el período estudiado. Para eso interesan hombres nuevos por definición, es decir los inmigrantes

14 Lo que explica que obras "'clásicas" de genealogía, como el Familias chilenas, de Guillermo de la Cuadra Górmaz, publicado en los años 1940, haya sido reeditado en 1982 y que diez años más tarde haya visto la luz, en el marco de las conmemoraciones del quinto centenario, el libro de Julio Retamal Favereau et al., Familias fundadoras de Chile. ;540-1600, Santiago, Ed. Zig-Zag, 1992.

15 Mario Góngora, Encomenderos y estancieros. 
españoles, cuyas trayectorias americanas, a pesar de sus particularidades individuales, presentan fuertes paralelos. Se puede observar en efecto, que aunque el cabildo de Santiago es un lugar de ejercicio del poder dominado por los criollos, como es natural, siendo la emanación del poder local urbano, los "peninsulares" tienen una relevancia relativa, ya que representan cerca del 20 por ciento, para redondear, del grupo estudiado. ${ }^{16}$

La primera constatación que se impone, y que confirma lo dicho anteriormente, es la importancia relativa de los inmigrantes vascos y navarros dentro de esta muestra, un poco más de la mitad del conjunto. Si a estos "vasco-navarros" se agregan los inmigrantes provenientes de las otras regiones de la cornisa cantábrica (asturianos, santanderinos, "montañeses"...), -asociación hecha por los mismos contemporáneos en el marco de cofradías por ejemplo- ${ }^{17}$ se está hablando de más de las tres cuartas partes del efectivo considerado. Esto no tiene que extrañar, pues coincide bastante bien con las cifras globales de la inmigración española a Chile durante el siglo XVIII, en las que los vasco-navarros constituían un poco menos de la mitad de todos los efectivos. ${ }^{18}$ Por otro lado, este fenómeno no es en absoluto una especificidad chilena, sino que es una tendencia global de la emigración española desde fines del siglo XVII. ${ }^{19}$

Ahora bien, el elemento que tiene gran importancia es la manera como estos inmigrantes, cualquiera que fuera su procedencia, lograron integrarse en la sociedad criolla con éxito, como su participación en el cabildo de Santiago lo demuestra.

${ }^{16}$ Este porcentaje corresponde a los 36 individuos llegados a Chile a fines del siglo XVíl y en el curso del siglo XVIII y que ocupan en algún momento de su vida un puesto de alcalde o de corregidor entre 1690 y 1790.

17 Los estatutos de fundación de la cofradía de Nuestra Señora de Aranzazu, de la nación vascongada, en Lima, incluían efectivamente a montañeses y santanderinos.

18 Según Arnold Bauer, Chilean rural society frotn the Spanish conquest to 1930- p. 16. cerca de 24.000 inmigrantes llegaron de España a Chile entre 1701 y 1810, 45 por ciento de los cuales venían de Navarra y del País Vasco.

${ }^{19}$ Mientras que la emigración española procedía mayoritariamente del sur de la península en el siglo XVI y XVII. desde finales del XVII se observa una inversión de la tendencia: los emigrantes ya no son andaluces, sino gallegos, asturianos, vascos y navarros. El período de más fuerte emigración norteña parece encontrarse en la última década del siglo XVII y en las primeras del siguiente, fenómeno ligado, en parte, a los períodos de crisis agraria cantábrica entre 1690 y 1710. Ver Ramón Lanza García. "'El contexto de los movimientos migratorios en Cantabria, siglos XV1I-XIX", Antonio Eiras Roel (ed.). La emigración española a Ultramar. 1492-1914. Madrid, Tabapress. 1991. 
En este proceso de integración, como en tantos otros de las sociedades del Antiguo Régimen, se debe prestar una particular atención a las redes en las que se encuentra inserto todo individuo. ${ }^{20}$ ya que éstas constituyen tanto importantes armas para el éxito social, como potentes elementos de auto-definición e identificación. En estas redes, la familia juega un papel que aunque no es exclusivo no es menos determinante.

El primer elemento que se encuentra al analizar los recorridos de un buen número de ediles peninsulares, es el clásico fenómeno del "mini-flujo" migratorio creado por todo migrante. En efecto, la mayor parte del tiempo la decisión individual de emigrar está motivada por el hecho de saber que en un determinado lugar un paisano o familiar puede ayudar al recién llegado, cuando no se trata simple y llanamente de responder al llamado de un pariente, un tío las más de las veces, que desea "remediar" a alguno de sus sobrinos dejandole el patrimonio o el negocio que hizo en América.

Es siguiendo este patrón de comportamiento como llegan a Santiago un buen número de sus alcaldes. Así, Juan de Lecaros, Navarro del Valle de Bertiz Aranaz llegado a Chile hacia 1675 (y alcalde de Santiago en 1693) es seguido por primos, sobrinos y parientes entre los que se encuentran José de Lecaros Egozcue, llegado con el ejército de Arauco precisamente al tiempo de la muerte de su primo (1694). Cerca de veinte años más tarde, un sobrino de José de Lecaros Egozcue, Pedro de Lecaros Berroeta, se encuentra en Santiago, en donde es alcalde en 1736. Considerando la cronología, es probable que este último haya arribado junto a otro pariente, Tomás Vicuña Berroeta, llegado a Chile en 1714 y alcalde de Santiago en 1733.

Otro pariente del primer Lecaros, originario a su vez del valle de Aranaz, llega a Santiago en los años inmediatamente posteriores a la muerte de Juan. Se trata de Santiago Larrain Vicuña, alcalde de la ciudad en 1707, el cual, siguiendo la misma lógica de solidaridad familiar, se convierte a su vez en el "embajador" de otros miembros de la familia entre los que se cuentan dos sobrinos que manda llamar. Martín de Larrain Vicuña, llegado a Santiago hacia 1744, y Francisco Javier de

${ }^{20}$ Juan Luis Castellano, Jean-Pierre Declieu (dir.), Réseaux, familles el pouvoirs dans le monde ibérique á la fin de l Anden Régime, París, CNRS Éditions, 1998. 
Errázuriz Larrain, alcalde de Santiago en $1756 .{ }^{21}$ Este Martín Larrain es el fundador de una numerosísima familia, conocida como la de "los ochocientos" durante la independencia de Chile por lo amplio y tentacular de su parentela.

Más ejemplos no harían sino confirmar la enorme importancia de este tipo de comportamientos entre los hombres del Antiguo Régimen. Debemos tener presente que todo individuo era educado con el sentimiento de ser parte de una "casa", noción mucho más amplia que la de linaje, en la que todos los miembros deben ser solidarios.

En efecto, si el linaje -conjunto de ascendientes y descendientes de una misma persona- insiste en los lazos de la sangre y del apellido común, la noción de casa hace referencia al conjunto de la parentela, así agnaticia como por afinidad. Parece necesario subrayar el hecho de que, a pesar del poder simbólico de la sangre, para explicar los lazos sociales es más importante considerar las relaciones entre los vivos, en el marco de la parentela extensiva, que la referencia al ancestro común propia del razonamiento genealógico del linaje.

El remedio de todos y cada uno de los miembros de una parentela le incumbe así a todos los que de una u otra manera tienen la posibilidad de ayudar. Tíos y tías se preocupan así por los oficios o los estudios de sus hijos, primos y sobrinos, por las dotes de las mujeres, de hacer todo lo que pueda ayudar, "adelantar" al grupo familiar. Esto no quiere decir que estas actitudes basten para explicar el lazo social, o que el hecho de formar parte de la misma familia explique todos los móviles de la conducta de un individuo o grupo de individuos: los odios más tenaces y los crímenes más horrendos ¿no tienen también como marco la familia? La costumbre y la presión social tienden sin embargo a la elaboración de una ética en la que el nepotismo no tiene nada de condenable, antes, es una virtud familiar deseada y esperada en los momentos de necesidad. ${ }^{22}$ En este caso, el sentimiento de "clan" -que logra durar dos a tres generaciones- es evidentemente una de las llaves para entender la exitosa integración de estos inmigrantes en el seno de la sociedad criolla.

21 José Toribio Medina. Diccionario biográfico colonial de Chile. Santiago, Imprenta Elzeviriana, 1906; Luis Thayer Ojecia, Navarros y vascongados en Chile, Santiago, Guillermo Miranda, 1904; Guillermo de la Cuadra Gormaz. Familias Chilenas, Santiago, Zamorano y Caperan, 1982 [1949].

22 Ver José María Imizcoz Beunza (dir.). Elites, poder y red social. Las elites del País Vasco y Navarro en la Edad Moderna. Bilbao. Universidad del País Vasco, 1996. 
Otro elemento fundamental caracteriza los recorridos de todos estos inmigrantes: todos, salvo raras excepciones, establecen alianzas matrimoniales con mujeres de la elite criolla. Más aún, una proporción significativa, un poco más de la mitad de aquellos inmigrantes, establece alianzas con criollas cuyo origen se remonta a la primera elite nacida de la conquista. La característica principal de estas mujeres, desde nuestro punto de vista, consiste en pertenecer a gmpos familiares que han logrado mantener, a través del tiempo, un status social que se puede medir gracias a la figuración de los miembros de la misma rama familiar en el ejercicio del poder local (en el cabildo) o en el prestigio de las alianzas matrimoniales que sus miembros siguen efectuando. El éxito individual de los inmigrantes se integra así en la continuidad de grupos familiares cuya antiguedad realza aún más el propio lustre: los apellidos cambian, los clanes permanecen.

Este es el caso del sevillano Fernando de Mendoza Mate de Luna, alcalde de Santiago en 1692, quien fuera gobernador del Tucumán de 1681 a 1686, fecha en la que se establece en Santiago al contraer matrimonio con doña María de Irarrazabal Zapata, perteneciente a una de las familias más poderosas e influyentes en el Chile de la época y cuyas ramificaciones se extendían a Lima, Madrid e Inglaterra. Doña María era la descendiente directa de don Francisco de Irarrazabal, ${ }^{23}$ rico encomendero de Santiago en el siglo XVI, su bisabuelo, quien después de casarse con una hija del contador de la casa de la contratación de Sevilla y comendador de la orden de Santiago, don Diego Ortiz de Zarate, había casado "noblemente" a todos sus hijos, actitud que sus descendientes habían mantenido después.

El recorrido de otro sevillano, Diego Calvo de Encalada, parece menos encopetado pero es tan revelador como el que se acaba de referir. Nacido en Sevilla en 1673 de familia hidalga (su padre era de la orden de Calatrava) don Diego pasa primero al Perú y luego a Chile a donde llega con las levas mandadas de Lima para la guerra de Arauco, como tantos otros españoles que alimentaban las filas del ejército permanente decretado por la Corona contra los rebeldes araucanos. En 1698, es capitán de una compañía de infantes y poco después es nombrado comisario general de caballería. Dos años más tarde contrae matrimonio en Santiago con

${ }^{23}$ Apellido euskera que los chilenos pronuncian Irarrázabal 
doña Catalina Chacón Carvajal, descendiente directa de Pedro de Miranda y de Diego Sánchez de Morales, conquistadores de Chile y encomenderos de Santiago y La Serena en el siglo XVI, importantes troncos de la sociedad criolla. Aún cuando no llevase ni el apellido Morales ni el apellido Miranda, doña Catalina pertenecía a una familia que no había decaído socialmente como las diferentes alianzas y títulos de sus antepasados lo demuestran (ver gráfico 1). En 1706, don Diego Calvo es electo alcalde de Santiago y en 1724 pasa a España con poderes del ejército. En la corte obtiene un hábito de Santiago y adquiere el título de marqués de Villapalma. $^{24}$

Si se considera el caso de la familia Lecaros, de la que ya se mostraron las ramificaciones, se encuentran de nuevo alcaldes de origen español que conjugan unos fuertes lazos con la familia peninsular, y una carrera local fundada sobre el prestigio de alianzas establecidas con la elite más enraizada y antigua (ver gráfico 2). Aquí, una vez más, las familias "nuevas" son, de cierta manera, la continuación de viejas estirpes con nuevos patronímicos.

Este proceso de fusión entre nuevos elementos y antiguos troncos familiares, como se puede apreciar en los gráficos, no es una característica del siglo XVIII, sino que resulta de una práctica totalmente connaturalizada e interiorizada por la elite criolla de Santiago. Durante todo el siglo XVII, en efecto, las familias de la elite criolla, a fin de garantizar su reproducción como grupo, adoptaron una doble actitud endogámica-exogámica, complementaria en su aparente contradicción. La primera "táctica" de los estratos altos consiste en una fuerte práctica endogámica social y familiar, esto es, casándose ya sea dentro de un estrecho círculo de familias de notables locales, ya sea al interior del propio grupo familiar. De manera paralela, estas mismas familias tratan por todos los medios de "captar" a todos los peninsulares llegados hasta este finís terrae -militares, funcionarios de las audiencias, gobernadores, etc. ${ }^{25}$ único medio de salir del modelo demográfico cerrado -y condenado a corto plazo- al que los llevaba la endogamia al interior de un universo social compuesto por un número muy limitado de familias ya ampliamente emparentadas. Así, a casi cada generación se van integrando, como

\footnotetext{
24 Por Real Cédula del 5 de octubre de 1728.

25 El matrimonio entre el antiguo gobernador del Tucumán. don Fernando Mendoza Mate de Luna y doña María de Irarrazabal, ya citado, es en este sentido un ejemplo típico de esta actitud.
} 


\section{Gráfico No. 1}

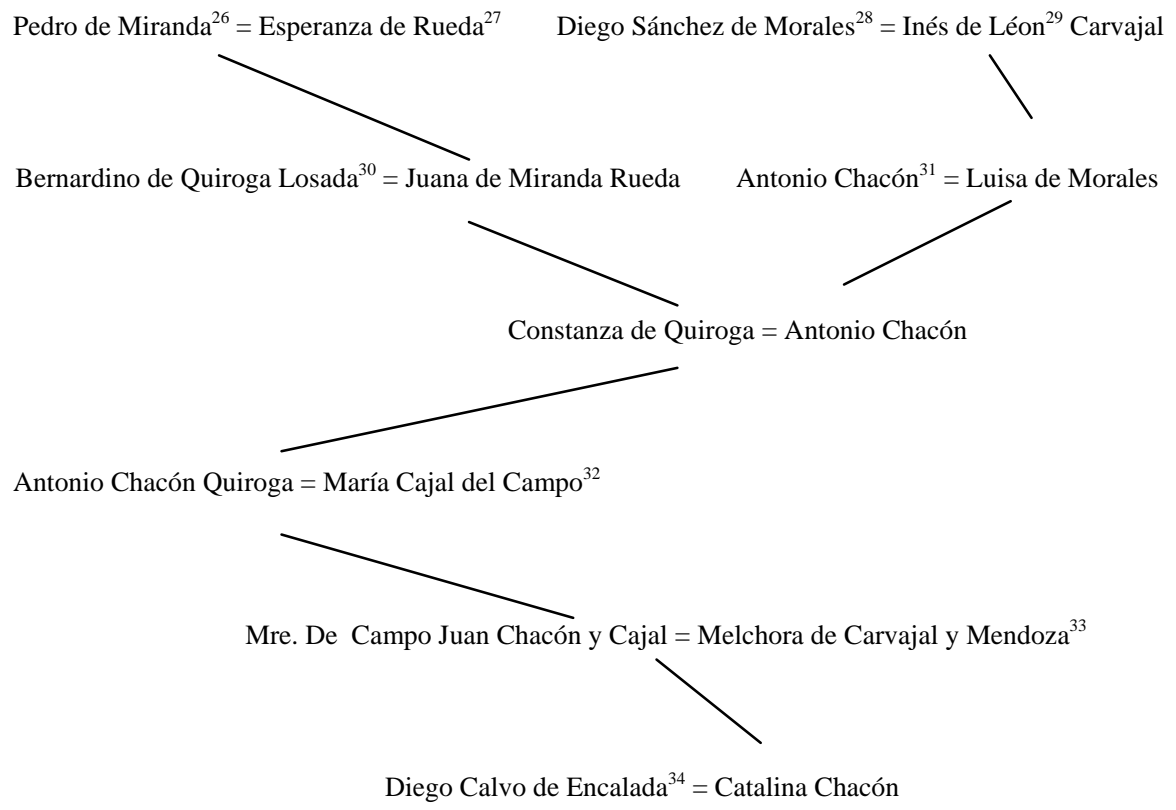

${ }^{26}$ Navarro, compañero de Valdivia y rico encomendero en Santiago. (Pueblo de Copequén). Entre otras funciones en el cabildo fue alcalde en 1556, 1559, 1561 y 1566.

${ }^{27}$ Natural de Epila (Zaragoza), sobrina de la mujer del adelantado Alderete.

${ }^{28}$ Natural de Soria. En el Perú, en 1534, participa en la expedición conquistadora de Chile y en las fundaciones de Santiago y de La Serena, en donde goza de la encomienda de Huasco. Alcalde de La Serena, en 1553 y 1561.

${ }^{29}$ Pariente de Alonso de Córdoba, rico encomendero de Santiago que pasó a España para traer su "casa" a Chile, en 1555.

${ }^{30}$ Hidalgo natural de Castiñeira, Galicia, hermano menor del señor de Cubillos, titular del mayorazgo del mismo nombre. Fue alcalde de Santiago en 1607.

${ }^{31}$ Natural de Fregenal de la Sierra hacia 1534. Llegado a Chile en 1560. Encomendero en Mendoza, corregidor de Cuyo, en 1574.

${ }^{32}$ Hija del alguacil mayor y regidor perpetuo de Santiago, Juan Cajal. y de la hija de un riquísimo comerciante de Santiago. Alonso del Campo Lantadilla. Juan Cajal era a su vez hijo del oidor del mismo nombre.

${ }^{33}$ Criolla, hija de Juan Carvajal y Mendoza, hijo a su vez del oidor de la Real Audiencia de Santiago (1623- 1632) y de Clara Flores de León, hija de Diego Flores de León, hidalgo madrileño, veterano de la guerra de Arauco. recompensado por el rey con una pensión de 1.800 ducados. Diego había hecho un buen matrimonio con una criolla de dote muy elevada. Fue asimismo corregidor de Concepción en diferentes oportunidades. El hermano de Clara Flores (y tío de Melchora) alcanzó el grado de maestre de campo y era caballero del orden de Santiago.

${ }^{34}$ Natural de Sevilla, caballero de la orden de Santiago, primer marqués de Villapalma. Alcalde de Santiago en 1706. 
en un tejido, nuevos elementos peninsulares que "renuevan la sangre" de la vieja elite, proporcionando nuevas ramas a los viejos troncos y manteniendo al mismo tiempo un fuerte lazo con la península, centro político y simbólico del imperio. En una sociedad nacida de la conquista, esta manera de "reactivar" el carácter hispánico de las elites, reviste una importancia simbólica crucial. Frente a las masas oscuras, la oligarquía criolla mantiene de esta manera su "blancura".

Ahora bien, considerando la naturaleza demográfica de los flujos migratorios peninsulares durante la mayor parte de la época colonial, este patrón de integración de los inmigrantes españoles implicaba, en la inmensa mayoría de los casos, un mercado matrimonial con una estructuración sexual fija, en la que España aparecía como una reserva de esposos para las jóvenes criollas, cuyas dotes eran tan cuantiosas cuanto la unión era deseada. El hecho de que la emigración peninsular fuera por lo esencial masculina ${ }^{35}$ conlleva así toda una serie de consecuencias que están lejos de ser meramente anecdóticas.

Primero que todo, este fenómeno explica el continuo cambio de apellidos dentro de los miembros de las elites políticas, como ya se apuntó más arriba con relación a la práctica matrimonial de los miembros del cabildo, y como se puede apreciar en los gráficos 1 y 2.

Por otro lado, la situación colonial -y la necesidad de mantener el status étnico de la familia- exacerba el papel tradicional de la mujer en la sociedad castellana como encamación literal del honor familiar. Toda familia de la elite debe procurar tener los medios de casar a sus hijas, o algunas de ellas, "conforme a su calidad", calidad expresada en el monto de la dote propuesta. Para atraer a hijos de oidores, a gobernadores o simplemente a hidalgos castellanos recién llegados es necesario hacer un importante esfuerzo económico, en el que participaban no solo tíos, tías y otros miembros de la familia, sino también las herencias legítimas de hermanos y de hermanas menores. En este caso no era raro que un "buen" matrimonio comprometiese el porvenir económico de los menores. El peculiar mercado

35 La única excepción a este modelo de emigración casi completamente masculina es la de mediados del siglo XVI en que las oportunidades que ofrecían sociedades recién fundadas -y lograda en su mayor parte la pacificación- atrajeron familias enteras de pobladores, hombres, mujeres y niños. Este modelo de emigración familiar no debía sin embargo perdurar de manera proporcionalmente significativa. 
matrimonial de las elites santiaguinas de los siglos XVII y XVIII implicaba una transmisión de paite importante de los patrimonios de suegro a yerno por intermedio de las mujeres. Los apellidos cambian pero los patrimonios se transmiten y se mantiene así la permanencia de buena parte de las elites originarias por vía femenina.

Por consiguiente, lo que fue tradicionalmente tomado como una prueba de inestabilidad de las elites no es sino la consecuencia de un método particularmente eficaz de perpetuación social, que se manifiesta en la proporción elevada (un poco menos de 60\%) de alcaldes y corregidores de Santiago en el siglo XVIII cuya filiación, las más de las veces materna, los hace descender directamente de las elites encomenderas del siglo XVI.

¿Qué habría pasado, por ejemplo, si la norma patrilinear dominante durante la mayor parte del siglo XVII y durante el XVIII hubiera sido precisamente matrilinear? Se encontraría sin duda que la gran mayoría de los vascos del siglo XVIII habrían tenido hijos cuyos apellidos continuarían siendo García de Cáceres, Pastene, o Miranda... De ser así, sin ningún cambio de la estructura de los linajes (sino solamente con un cambio de las normas de transmisión del apellido), la antroponimia mostraría un caso de gran estabilidad!

Cabe subrayar en este sentido, que el estudio de la estructura de la elite de los notables de Santiago en el siglo XVIII a través de su figuración en el cabildo de la ciudad, revela un modelo de reproducción que parece ser exactamente el mismo que en el siglo XVII. La única diferencia entre ambas épocas es que los flujos migratorios peninsulares cambian, y si durante el XVI y buena parte del XVII fueron preferentemente los hombres del sur de la península los que se exiliaron, a partir de fines del XVII son los hombres del norte, de toda la cornisa cantábrica, quienes toman la ruta de América. La ola vasco-navarra que tan presente tenían los historiadores chilenos del siglo pasador-interés particular que todavía se puede percibir de manera residual o "fósil" en los historiadores chilenos de la actualidad-

\footnotetext{
${ }^{36}$ A tal punto que el gran historiador chileno Benjamín Vicuña Mackenna en su obra en tres partes Oríjenes de las familias chilenas, dedica una completa a los "vizcaínos", la primera, y las otras dos a los apellidos de origen no hispánico... olvidando las familias de origen castellano! Ver Benjamín Vicuña Mackenna, Oríjenes de las familias chilenas. I: Los vizcaínos; II: Los portugueses. Los holandeses. Los italianos. Los malteses y griegos. Los nombres trocados; III: Los franceses. Los irlandeses. Santiago, G. E. Miranda, 1903.
} 


\section{Gráfico No. 2}
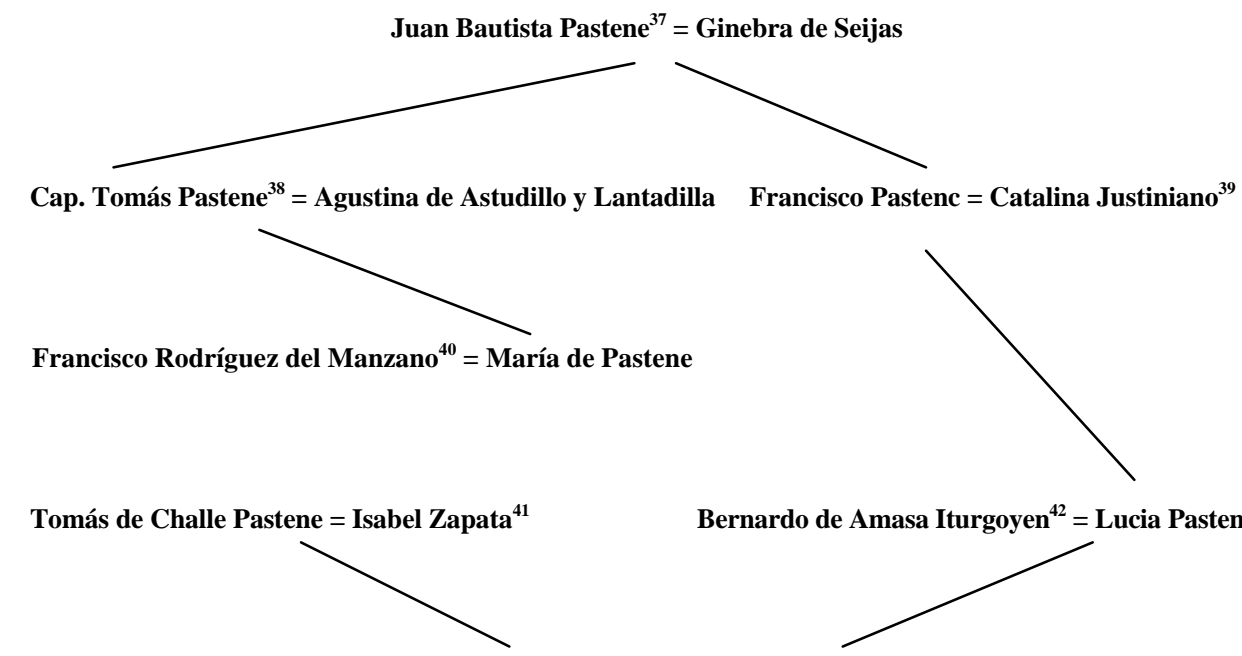

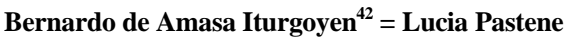

Capitán Alonso de Ovalle ${ }^{43}=$ María Amasa Iturgoyen

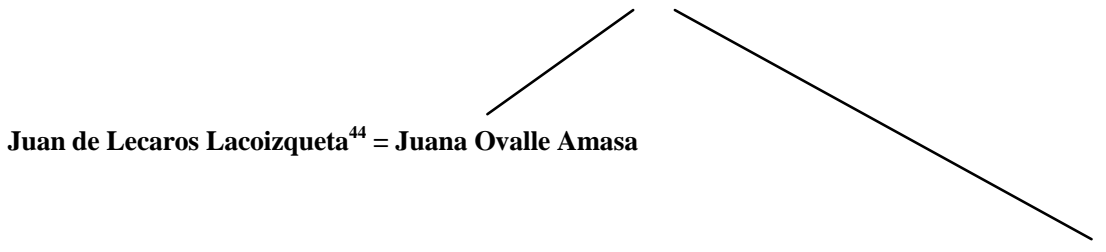

José de Lecaros Egozcue ${ }^{45}=$ María Ovalle Amasa

${ }^{37}$ Conquistador genovés. Encomendero de Tagua tagua. Mapocho y Puangue (Santiago). Cinco veces regidor del cabildo de Santiago, y su alcalde en 1564.

${ }^{38}$ Heredero de las encomiendas de su padre. Alférez real en 1575, alcalde de Santiago en 1580, 1587, 1593. Se casa en Sevilla en 1567. Su mujer parece ser pariente del rico mercader de comienzos del siglo XVII Alonso del Campo Lantadilla.

${ }^{39}$ Hija de Juan Ambrosio Justiniano. maestre de navio natural de Genova.

${ }^{40}$ Hidalgo natural de Salamanca, alcalde de Santiago en 1613. 1619, 1625 y 1640.

${ }^{41}$ Criolla, hija de Jerónimo Zapata, natural de Palencia, venido a Chile para heredar los bienes de su tío, Antonio de Zapata, socio del rico encomendero Alonso de Córdoba. Su mujer era natural de Valdepeñas y pariente de Alonso de Córdoba. Jerónimo de Zapata fue alcalde de Santiago en 1606 y 1621.

${ }^{42}$ Natural de Alegría (Guipúzcoa), pasa al Perú como soldado, y a Chile como capitán de infantería en 1621. Asciende a maestre de campo en 1626. Pasa a España de donde vuelve en el séquito del marqués de Baides, gobernador de Chile (1638). Alcalde de Santiago en 1639 y 1649. Corregidor de Santiago en 1642.

${ }^{43}$ Alcalde de Santiago en 1665.

${ }^{44}$ Natural de Narbarte. Navarra, llega a Chile en 1675. Alcalde de Santiago en 1693.

${ }^{45}$ Natural de Ciga. valle del Baztán. llegado a Chile con el ejército de Arauco entre 1695-1700. 
es un elemento más que une a Chile con la historia del resto de la América española, que en su conjunto conoció este mismo movimiento. La relativa "vasquización" de la oligarquía santiaguina no tiene así nada de asombroso sino que es el resultado lógico de la combinación de la dinámica de las elites criollas y de los flujos demográficos peninsulares.

Sin embargo, si el comportamiento tradicional de la oligarquía de Santiago explica la abundancia de patronímicos vascos y del norte de la península en general, el hecho de que estos apellidos hayan figurado a partir del XVIII en la cúspide del prestigio y de la jerarquía social parece efectivamente una novedad con respecto a los periodos anteriores.

La explicación tradicionalmente avanzada, y que tiende a olvidar que los descendientes de los inmigrantes del norte de la península tenían madres y familias criollas, es que esta ola tenía ciertas peculiaridades sociales, como un mayor contacto con el mundo del comercio, y una cierta austeridad, rasgo de carácter que les habría permitido conservar su situación de preeminencia. Los hombres del norte son así para la historiografía tradicional chilena lo que los protestantes para la sociología weberiana.

Quizás sea posible explicar esta particularidad si consideramos las mutaciones globales que vive la sociedad y la economía chilenas durante el siglo XVIII. Durante este periodo, la población conoce un crecimiento sostenido, lo que lleva a los gobernadores José Manso de Velasco (1737-1744) ${ }^{46}$ y Domingo Ortiz de Rozas (1745-1754) a organizar y financiar parcialmente la fundación de nuevas ciudades en el valle central. Es así como entre 1740 y 1754 son creadas ciudades -en realidad pueblos- como San Felipe, Los Angeles, Cauquenes, Talca, San Fernando, Melipilla, o Rancagua... Este crecimiento de la población implica una ampliación del mercado potencial, pero sobre todo, la abundancia de mano de obra barata y móvil, como lo explicaba en 1778 don José de Ureta, corregidor de la provincia de Melipilla, "los vecinos y naturales de esta provincia, unos tienen tierras propias, otros arrendadas y otros nada. Y muchos ociosos por no tener en qué trabajar y otros trabajar a jornal. El número de unos y otros en cada partido es inaveriguable, por motivo

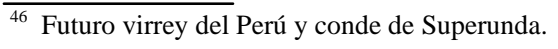


que en unas ocasiones tienen en que trabajar y otras no; $y$ andar de partido en partido los que no tienen tierras propias." ${ }^{47}$ En estas circunstancias, el bajo nivel de los salarios permite incrementar las márgenes beneficiarias de la actividad agrícola.

Sin embargo, el principal incentivo de la economía de la zona central viene sin lugar a dudas fundamentalmente del exterior. En efecto, las dificultades agrícolas que conoce el Perú a fines del siglo XVII le dan unas proporciones sin precedentes a las exportaciones de trigo chileno hacia el Callao. Entre 1694 y 1751, éstas pasan de 728 toneladas anuales a $9.328 .^{48}$ Durante el período comprendido entre 1680 y 1739 este fuerte incremento de la demanda provocó un aumento general de los precios de toda la producción agrícola, vegetal y animal, y por consiguiente, del precio de la tierra. Aún cuando durante el período siguiente los precios tuvieron tendencia a bajar, esta baja de los precios de la producción agropecuaria (trigo, sebo, cueros) era compensada por el volumen de las exportaciones, que siguió siendo muy importante. ${ }^{49}$

Como se ve, esta coyuntura económica es particularmente favorable para los grandes propietarios de tierras agrícolas. Y justamente, son los mismos grupos que acaparan el poder municipal los que concentran la posesión de la tierra, la fuerza de trabajo indígena, esclava o asalariada y las riendas del comercio. Producción y distribución se hallan así entre sus manos, lo que hace de ellos los grandes beneficiarios del formidable aumento de la demanda de trigo por parte del mercado limeño. El siglo XVIII es, por consiguiente, una época de acumulación de fortunas a un nivel desconocido anteriormente. Con el desarrollo económico de la colonia, las fortunas criollas acceden a formas de consolidación de los patrimonios que les estaban vedadas en la práctica, hasta entonces, por las costas que implicaban. Vínculos, mayorazgos y títulos de nobleza ven el día en Chile casi exclusivamente durante el siglo XVIII, en circunstancias en que en otras colonias más pudientes (México, Perú) las grandes familias criollas no esperaban tanto tiempo para invertir sus fortunas en estrategias económico-simbólicas de tipo

\footnotetext{
$\overline{47}$ Francisco de Solano (ed.). Relaciones económicas del Reino de Chile. 1780. Madrid, CS1C. 1994.

${ }^{48}$ Marcello Carmagnani. Les mécanismes de la vie économique dans une sociélé coloniale: Le Chili (1680-1830), París, SEVPEN. 1973, p. 53.

${ }^{49}$ Ibid., p. 54.
} 
"superior". Es así como durante este período Juan de la Cerda, Andrés de Toro Mazote, Don Antonio de Irarrazabal, Santiago de Larrain, Sebastián de Lecaros, Juan Nicolás de Aguirre, Francisco García Huidobro, Domingo de Valdés, Pedro Fernández Balmaceda, Francisco Antonio Ruiz de Tagle, José Miguel de Prado y Covarrubias, Rosa Rojas Cerda, y Mateo de Toro Zambrano fundaron mayorazgos sobre sus propiedades. ${ }^{50}$ De todos estos individuos, sólo dos pertenecían a familias que no figuraron durante este período entre los miembros del cabildo, y siete eran o provenían de hombres nuevos llegados a partir de finales del siglo XVII. Como reza el formulario de fundación, "para que vuestra casa quede, finque y se conserve..." ${ }^{51}$, el mayorazgo aparece aquí como una manera de inscribir el apellido en el tiempo, novedad en la sociedad chilena colonial, elemento que me parece fundamental. El mayorazgo permite una transformación mayor en el Chile del siglo XVIII pues excluye, en la inmensa mayoría de los casos, a las mujeres, aquellas precisamente que habían sido las protagonistas imprescindibles de la transmisión patrimonial hasta entonces.

Este cambio traduce la nueva fuerza económica de la elite de Santiago, a la vez que su crecimiento demográfico. La transmisión patrilinear del patrimonio supone en efecto la existencia de un mercado matrimonial femenino suficiente para asegurar la perpetuación del apellido sin desmedro social. Esto es sin lugar a dudas la consecuencia del desarrollo demográfico de la elite de Santiago durante el siglo XVIII.

En definitiva, el mayorazgo, y en cierta medida la adquisición de títulos de nobleza, -títulos que suelen ser obtenidos por personas pertenecientes a las mismas familias que se han venido nombrando- ${ }^{52}$ permiten unir de manera

50 Ver Domingo Amunátegui Solar, La sociedad chilena en el siglo XVIII. Mayorazgos y títulos de Castilla Santiago, Imprenta Barcelona, 1901.

51 Citado por Jean-Pierre Dedieu, "Familles, majorats, réseaux de pouvoir. Estrémadure, XVe-XVlIIe", Juan Luis Castellano, Jean-Pierre Dedieu, p. 116.

52 Como por ejemplo el conde de Sierra Bella, título de la familia Mesía de Torres; el título de marqués de Montepío perteneciente a la familia Aguirre; el título de conde de la Conquista, perteneciente a los Toro Zambrano; el título de marqués de Larrain, de la familia Larrain y Guzmán; el título de marqués de Villapalma de Encalada, perteneciente a la familia Calvo de Encalada: en cada uno de estos casos se trata de familias de cabildantes y de inmigrantes recientes. 
íntima apellido y patrimonio, variables que hasta el siglo XVIII no parecen ir obligatoriamente unidas. No es entonces a un reemplazo de las elites al que asistimos sino a la consagración de un ideal social.

Los mecanismos comprobados y particularmente eficaces que se han visto en acción le permiten así a la elite de Santiago de Chile reproducirse física y económicamente, y conservar al mismo tiempo una imagen de sí misma. Estos comportamientos que se remontan al siglo XVII e incluso al XVI son los que posibilitan la constitución de una oligarquía muy homogénea, grupo que se define así mismo como "aristocrático"', y que logra imponer su modelo social al resto de la sociedad. Durante el siglo XVIII este grupo logra darle a su ideal aristocrático su expresión más acabada: el auge económico permite, en efecto, la vinculación de propiedades gracias a la fundación de mayorazgos y a la adquisición de títulos nobiliarios, que unen patrimonio y nombre, lo que graba en la memoria social, y de manera extremadamente duradera, el prestigio -y el poder económico- asociado a ciertos linajes. Los apellidos antiguos como Toro Zambrano, Irarrazabal, de la Cerda, producto de la sedimentación de los siglos XVI y XVII se fijan junto con los más modernos, Lecaros, Larrain, Errazuriz, Zañartu, Calvo Encalada, y marcan con su cuño no sólo el siglo XVIII sino también buena parte de la historia del Chile republicano. ${ }^{53}$

Lazos familiares renovados a cada generación con la "madre patria", educación en la Compañía de Jesús, viajes a la corte virreinal e incluso a España -lo que no deja de recordar el viaje a Italia de las elites aristocráticas europeas renacentistas, especie de vuelta a las raíces culturales- son características de la élite, a las que se tiene siempre que agregar el ejercicio de cargos en la administración imperial y sobre todo, como se ha visto, en el cabildo, eje imprescindible para enraizar en la esfera local a estos grupos "cosmopolitas". Todos estos elementos forman parte del desenvolvimiento típico de la vida de los hombres de la elite y conforman una especie de modelo aristocrático considerado como el ideal al que todos deben tender. Este modelo

\footnotetext{
Ver Mary Lowenlhal Felstiner, "Kinship Politics in the Chilean Independence Moment",Hispanic American Histórical Review, vol. 56, No. 1, 1976; Diana Balmori, Robert Oppenheimer, "Family Chistas: Generational Nucleation in Nineteenth-Century Argentina and Chile",Comparative Studies in Society and History, vol. 21, No 2, 1979.
} 
de reproducción de la elite es sin duda un elemento fundamental para entender no sólo los siglos de la época colonial sino incluso las características del movimiento de independencia y la subsiguiente construcción de una república independiente. En efecto, la fuerte dependencia cultural y demográfica con respecto al santuario peninsular parece llevar lógicamente a una gran dificultad para elaborar, para conceptual izar, la idea nacional. La constitución de redes de familias que comparten una serie de valores y de comportamientos, que están relacionadas a nivel de todo el territorio del Chile colonial, y a su vez con España e incluso con otros países europeos, que poseen grandes propiedades territoriales y las riendas del comercio, parecen separarlas del resto del "común". Esta aparente división, elemento que constituye el nudo del pensamiento oligárquico de las dos sociedades paralelas a las que se hacía referencia al comenzar esta contribución, evoca mutatis mutandis, la difícil gestación de la idea de Nación en la Francia del siglo XVIII, que a partir de una sociedad estamental produce la idea de una sola comunidad, un solo pueblo. $^{54}$ Pero ¿cómo hacer aceptar a los miembros de la oligarquía chilena que formaban un mismo pueblo, una misma comunidad, con las chinas y peones de sus casas y de sus haciendas? Las peculiares definiciones de la nación y del poder político ${ }^{55}$ tal como surgen de este problema, en buena parte insoluble, parecen enraizarse en el modelo de autodefínición lentamente elaborado por la oligarquía criolla chilena a través de dos siglos y medio de integración -y simbiosis- con el imperio español.

\footnotetext{
54 Este paralelo es tanto más pertinente por cuanto los acontecimientos de Francia fueron después ampliamente instrumental izados por la élites de la América española.

55 En particular el hecho de definir la nación como una comunidad soberana, cuyo poder debía ser ejercido según la voluntad de la maior pars, principio revolucionario que la élite traduce como la mejor parte, i sanior pars.
} 